\title{
Severity Analysis of Macular Edema using Random Tree Classifier
}

\author{
Deepthi K Prasad* ${ }^{1}$, Vibha $L^{2}$, Venugopal $K \mathbf{R}^{3}$ \\ ${ }^{1}$ Full Time Research Scholar, Department of CSE, B N M Institute of Technology, \\ Bangalore, Karnataka, India \\ ${ }^{2}$ Professor, Department of CSE, B N M Institute of Technology, \\ Bangalore, Karnataka, India \\ ${ }^{3}$ Principal, UVCE, Bangalore University, \\ Bangalore, Karnataka, India
}

\begin{abstract}
:
Macular edema ensues when there is abnormal pile-up of fluid and results in swelling of the macula part of the retina. It is commonly associated with diabetes. It can be diagnosed by identifying exudates in the retinal images. In the proposed work, macular the retinal image is pre-processed, enhanced and segmented using morphological operations. The optic disc and macula are segmented. Various statistical features are extracted. Optimal features are selected using Haar wavelets. The selected features are classified using Random tree classifier to detect the severity of the disease in to three stages namely, normal, mild and critical. The accuracy obtained is $98.4 \%$.
\end{abstract}

Keywords: Macular edema, exudates, macula, optic disc, Haar wavelets, Random tree, Classification.

\section{Introduction}

Diabetes mellitus is a chronic disorder which occurs when the pancreas do not produce adequate insulin in the body. According to the WHO global report [16] there were 1.5 million deaths in the world caused only due to diabetes. If the blood sugar levels are not controlled properly, the retina may get damaged, resulting in partial or total vision loss. Diabetic retinopathy (DR) is the injury to the retina caused due to the damaged blood vessels. Studies prove that the percentage of retinopathy in people with diabetes is $\mathrm{s} 35 \%$ [1]. If DR is not detected at early stage, the aqueous fluid leaked by the blood vessels accumulates in the macula region of the retina. This causes the macula to swell and is referred as macular edema (ME). ME is the most common reason for vision loss among people with DR.

ME, if left untreated can result in serious vision loss and even blindness. The most simple and effective way of detecting ME is by checking for the presence of Exudates in the retinal region. Exudates occur when the capillaries leak lipids and plasma. The surplus collection results in the inflammation of the retina. The image with macular edema is given in Figure 1.

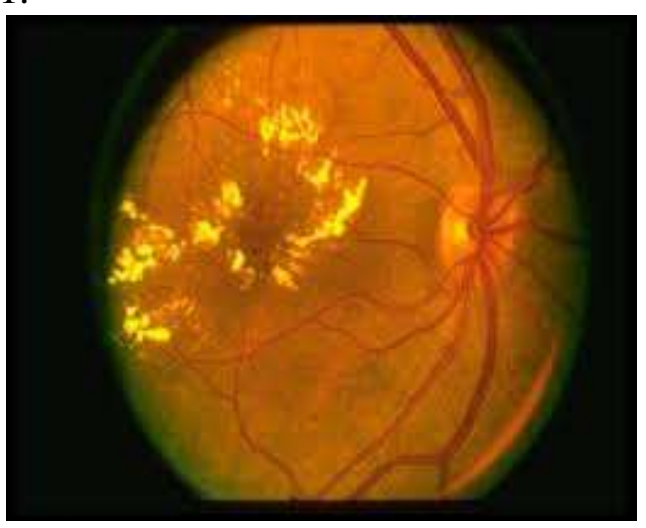

Figure 1. Retinal Image with Macular Edema

Macular edema usually doesn't show any visible symptoms when it develops. Blurred vision and loss of sharp sight are the major symptoms once the disease starts progressing.

Motivation: Macular edema is a retinal bruise caused due to repercussion of DR. It may lead to vision loss, if not diagnosed at an early stage. Manual detection of this disease is not adaptable due to the shortage of experts or equipment in rural areas. A computerized ME detection system can overcome these limitations and refer only those 
cases which need immediate attention of a doctor.

Contribution: The proposed work takes the retinal input images as input, preprocesses, segments the exudates, extracts the required features, selects the relevant features and classifies them based on the severity as normal, mild or critical stages using the Random tree classifier.

The outline of the work is as follows. Earlier methods in the ME analysis is described in Section 2. Section 3 describes the architecture of the ME detection system. The next section discusses the results obtained and comparative study. The last section presents the conclusion and future enhancements.

\section{Literature Review}

J J Kumar and C G Ravichandran [1] developed a technique for identification and grading of ME from retinal images. Hard exudates are detected using extreme learning machine classifier. The severity of $\mathrm{ME}$ is assessed using the regional properties of hard exudates in the retinal image.

R S Rekhi et al. [2] developed a robust method to segment hard exudates from retinal fundus images. The geometrical shape and orientation features of exudates are considered for accurately segmenting exudates resulting in an overall accuracy of $91 \%$ for 189 fundus images from DIARETDB1 and MESSIDOR databases.

ME grading based on feature learning approach and deep neural networks was proposed by B. Al-Bander et al. [3]. The algorithm was tested on MESSIDOR database and an accuracy of $88.8 \%$ was obtained using this method.

Lim et al. [5] presented an automated approach for classifying diabetic macular edema. The macula area is marked and fovea is localized based on ETDRS rule. "Marker controlled watershed transformation" is employed to separate exudates from the macula regions to classify as normal, stage 1 and stage 2 . An accuracy of $85.2 \%$ is obtained with this method for MESSIDOR data set.

S. Vasanthi and Wahida Banu [6] suggested a technique for automatic segmentation of hard exudates from retinal fundus images using "Adaptive Neuro Fuzzy Inference System" and "Extreme Learning Machine classifier". They reported an accuracy of $96.49 \%$ for both the classifiers.
A hybrid classifier system was proposed by U. R. Acharya et al. [7] using discrete wavelet, Radon and discrete cosine transformations. Supervised hybrid classifiers are used to classify using significant features. The accuracy obtained for the MESSIDOR database is $97 \%$.

Akram et al. [8] presented an intelligent system for detecting ME using a Gaussian mixture model and detailed feature set. A hybrid classifier using the combination of Gaussian mixture model and the SVM classifiers is proposed. They obtain an accuracy of $96.8 \%$ for the standard retinal image databases.

Periera et al. [9] proposed a method for segmenting exudates using "ant colony optimization" method. Performance was experimented with an online database and the authors have claimed that performance outweighs the result of Kirsch filter in exudates detection.

B. Harangi and A. Hajdu [10] developed an automated exudates detection method using a three stage approach which included candidate extraction, precise contour segmentation and candidate labeling. Naïve Bayes classifier combined with an adaptive boosting technique is used for classification.

Mishra and Ratha [14] inferred that attribute selection based random tree classifier can produce better accuracy of classification compared to random forest classifier. They had tested it on publicly available microarray datasets.

\section{The Proposed System}

\subsection{Overview of the proposed system}

The proposed system comprises of a data aggregation step where the input images are received from a standard retinal image database. The images are pre-processed to make it suitable for extracting the exudates by using various morphological functions. Exudates are segmented and macula region is isolated. Various statistical features are extracted from the grey scale image as well as exudates segmented image. Optimal features are selected using Haar wavelets. Classification is done using Random tree classifier. The architecture of this system is given in Figure 2. 


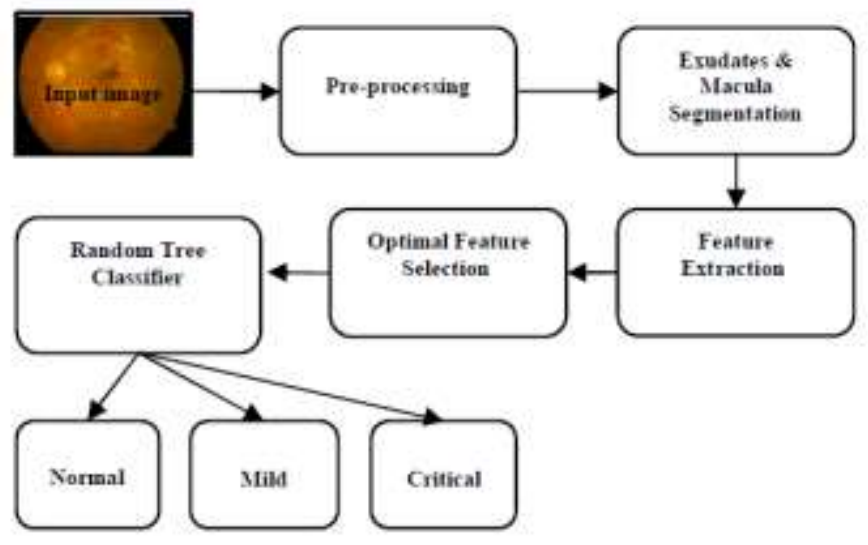

Figure 2. Architecture of the ME Detection System

\subsection{Image Pre-processing}

The input images come from varying databases and are converted to a size of $373 \times 565$ pixels but the aspect ratio is maintained. The channels of the image namely, red, green and blue are extracted and analyzed. Green indexed image is chosen as it preserves contrast feature with the background. Histogram equalization is performed to improve the image contrast.

The regions of interest in the proposed system are exudates and macula. Macula is the darkest region in the retinal fundus image which helps in achieving sharp vision. The histogram equalized image is transformed to binary image. Any noise present in the image is removed using the median filter. This image is complemented to detect macula.

The optic disc is the brightest part of the retinal image and segmented by setting the threshold intensity of the image. The intensity of the detected regions is maximized and morphological functions are used to fill the gaps in the image. The closed image is converted to binary image to segment the optic disc.

The hard exudates are the yellow flecks found in the retinal image. Morphological close operation using octagon shaped structuring element is used. To highlight the edges of the retinal image, Sobel operator is used. Morphological close operation using a circular structuring element is used and median filter is used to remove the noise to segment exudates.

\subsection{Feature Extraction}

The exudates segmented image is considered and the number of exudates regions is counted. The area occupied by the exudates in each region is computed. The various statistical features are extracted using [11]. The features extracted are kurtosis, entropy, mean, third moment and gray level co-occurrence matrix[11].

\subsection{Feature Selection}

Optimal features have to be selected for accurate classification. The proposed work uses Discrete Wavelet Transform (DWT) using Haar wavelets [12]. Haar wavelets have been extensively used in feature selection because of its low computing requirements [12]. It is the easiest, faster than other methods [13].The features are converted to discrete wavelets and then fed to the Random tree classifier.

\subsection{Classification using a Random tree classifier}

Random tree is a classification algorithm based on supervised classification. They were developed by Leo Breiman and Adele Cutler. It uses the concept of bagging to build a decision tree for constructing a random set of data [14]. This algorithm can handle both classification and regression types. Random trees are a collection of tree predictors. They are amalgamation of two important algorithms used extensively in machine learning namely, single model decision trees and random forest concept. Algorithm for random tree classification is described in Table 1.

Table 1. Generalized Algorithm for Random Tree Classifier

Input: feature vector $V$

Output: class labels

Step 1: Get the input feature vector from the feature set

Step 2: Build $N$ un-pruned random trees from the feature vector $V$

Step 3: Randomly select $p$ predictors at each node. From these select the best split

Step 4: Save the tree

Step 5: Classify each input attribute vector from V with every tree formed in the forest

Step 6: Identify the class label that obtained maximum number of votes

Step 7: output the class label that has maximum votes 


\section{Results Analysis}

\subsection{Results of Image Pre-Processing}

The results of pre-processing the retinal fundus images are discussed below. Figure 3a. shows the histogram equalized image and Figure $3 b$ shows the macula detected image obtained as a result of methods explained in Section 3.2.

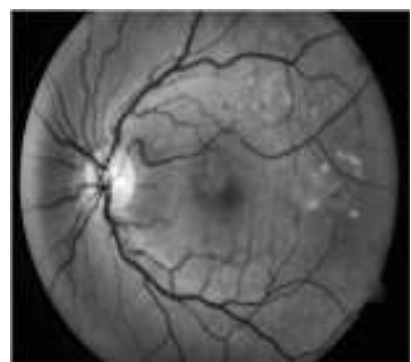

(a)

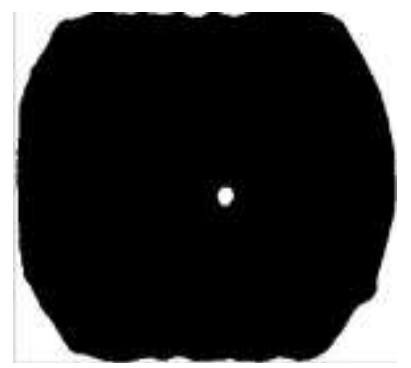

(b)
Figure 3 a. Histogram equalized image b. macula detected image

The optic disc is obtained from the green channel image using various morphological operations described in section 3.2. The results are depicted in Figure $4 \mathrm{a}$ and $4 \mathrm{~b}$ respectively

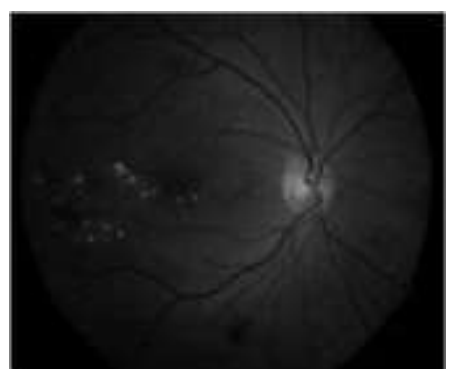

(a)

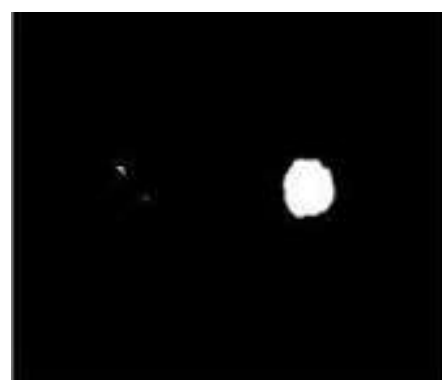

(b)
Figure 4 a. Green channel image b. Optic disc detected image

The segmentation of hard exudates from histogram equalized image is depicted in Fig 5. Figure 5a shows the histogram equalized image and $5 \mathrm{~b}$ represents the segmented exudates image respectively

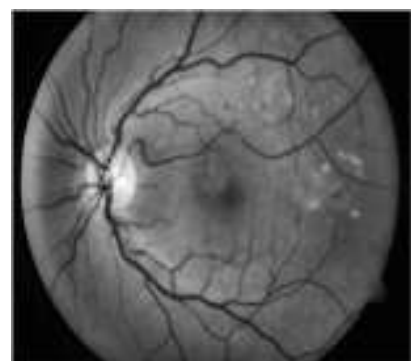

(a)

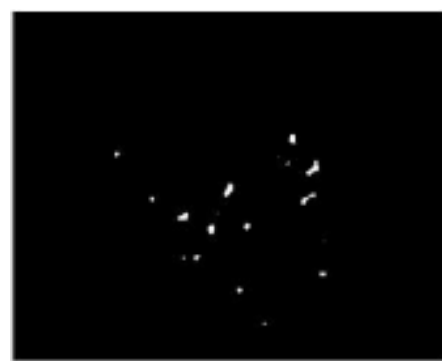

(b)
Figure 5a. Histogram equalized image b. Segmented Exudates

\subsection{Performance Analysis}

Classification of the fundus images were performed using random tree classifier. A total of 600 images were randomly selected from the MESSIDOR dataset [15]

The proposed method classifies the given retinal images into one of the three classes namely, normal, mild or critical. Hence the confusion matrix is a $3 \mathrm{X} 3$ matrix as shown in Table 2.

Table 2. Confusion matrix for random forest classifier

\begin{tabular}{|l|l|l|l|}
\hline Class & Normal & Mild & Critical \\
\hline Normal & 344 & 1 & 0 \\
\hline Mild & 2 & 159 & 0 \\
\hline Critical & 0 & 4 & 90 \\
\hline
\end{tabular}

The accuracy for normal, mild and critical classes obtained were $99.42 \%, 96.95 \%$ and $100 \%$ respectively. The overall accuracy for all the three classes is $98.83 \%$ which is very high and stable.

The proposed method showed outstanding results compared to earlier methods in terms of increased accuracy of detection. The graphical representation of the performance analysis is depicted in Figure 6.

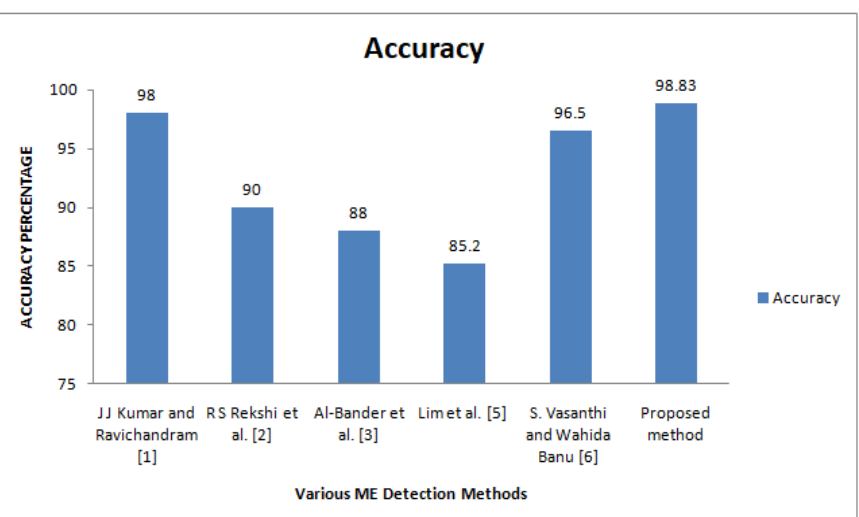

Figure 6. Performance Analysis of ME Detection Methods

\section{Conclusion}

Macular edema is the most common reason for vision impairment in people with uncontrolled sugar levels. This work proposes a method for severity identification of macular edema using random tree classifier. A total of 600 images are considered from MESSIDOR database. Various morphological operations are performed on the input fundus images to segment macula, optic disc and 
exudates. The statistical features are extracted and discrete wavelet transformation using Haar wavelets is used for better feature selection. The selected features are fed to the random tree classifier to partition the input images into normal, mild or critical stages. The proposed work resulted in an accuracy of $98.8 \%$ which outweighs the earlier methods for severity detection and classification in the literature.

As a future work, heterogeneous databases may be incorporated and better image pre-processing techniques could be used for handling varying image data sources.

\section{References}

[1] J. J. Kumar and C. G. Ravichandran, "Macular Edema severity detection in colour fundus images based on ELM classifier," 2017 International Conference on I-SMAC (IoT in Social, Mobile, Analytics and Cloud) (ISMAC), Palladam, 2017, pp.926-933. doi: 10.1109/I-SMAC.2017.8058316

[2] S. Rekhi, A. Issac, M. K. Dutta and C. M. Travieso, "Automated classification of exudates from digital fundus images," 2017 International Conference and Workshop on Bioinspired Intelligence (IWOBI), Funchal, 2017, pp.1-6. doi: 10.1109/IWOBI.2017.7985527

[3] Al-Bander, Baidaa; Al-Nuaimy, Waleed; AlTaee, Majid A.; Williams, Bryan M.; and Zheng, Yalin. Diabetic Macular Edema Grading Based on Deep Neural Networks", In: Chen X, Garvin MK, Liu J, Trucco E, Xu Y editors. Proceedings of the Ophthalmic Medical Image Analysis Third International Workshop, OMIA 2016, Held in Conjunction with MICCAI 2016, Athens, Greece, October 21, 2016. 121128. https://doi.org/10.17077/omia.1055

[4] Lim, Sin Ting \& Zaki, W \& Hussain, Aini \& L. Lim, S \& Kusalavan, Shivambiga, "Automatic classification of diabetic macular edema in digital fundus images", 10.1109/CHUSER.2011.6163730A, 2011.

[5] Vasanthi, S \& Wahida Banu, R.W, "Automatic segmentation and classification of hard exudates to detect Macular Edema in fundus images", Journal of Theoretical and Applied Information Technology. 66. 684-690, 2014.

[6] Valverde C, García M, Hornero R, LópezGálvez MI. "Automated detection of diabetic retinopathy in retinal images. Indian Journal of Ophthalmology", 2016;64(1):26-32, 2016.
[7] U. Rajendra Acharya, Muthu Rama Krishnan Mookiah, Joel E.W. Koh, Jen Hong Tan, Sulatha V. Bhandary, A. Krishna Rao, Yuki Hagiwara, Chua Kuang Chua, Augustinus Laude, "Automated diabetic macular edema (DME) grading system using DWT, DCT Features and maculopathy index", Computers in Biology and Medicine, Volume 84, 2017, Pages 59-68, ISSN 0010-4825, 2017. https://doi.org/10.1016/j.compbiomed.2017.03. 016

[8] M. Usman Akram, Anam Tariq, Shoab A. Khan, M. Younus Javed, "Automated detection of exudates and macula for grading of diabetic macular edema", Computer Methods and Programs in Biomedicine, Volume 114, Issue 2, Pages 141-152, ISSN 0169-2607,2014, https://doi.org/10.1016/j.cmpb.2014.01.010

[9] Carla Pereira, Luís Gonçalves, Manuel Ferreira, "Exudate segmentation in fundus images using an ant colony optimization approach", Information Sciences, Volume 296, 2015, Pages 14-24, ISSN 0020-0255, https://doi.org/10.1016/j.ins.2014.10.059

[10]Balazs Harangi, Andras Hajdu, "Automatic exudate detection by fusing multiple active contours and regionwise classification", Computers in Biology and Medicine, Volume 54, Pages 156-171, ISSN 0010-4825, 2014, https://doi.org/10.1016/j.compbiomed.2014.09. $\underline{001}$

[11]D. K. Prasad, L. Vibha and K. R. Venugopal, "Early detection of diabetic retinopathy from digital retinal fundus images," 2015 IEEE Recent Advances in Intelligent Computational Systems (RAICS), Trivandrum, 2015, pp. 240245.

doi: 10.1109/RAICS.2015.7488421

[12]P. Porwik, A. Lisowska, "The Haar Wavelet transform in Digital Image Processing: Its Status and Achievements," Machine Graphics and Vision, Poland, 2004

[13]Subramani, Prabakaran, Rajendra Sahu, and Shekhar Verma. "Feature Selection Using Haar Wavelet Power Spectrum." BMC Bioinformatics 7 (2006): 432. PMC. Web. 10 Feb. 2018.

[14]Ajay Kumar Mishra \& Bikram Keshari Ratha, "Study of Random Tree and Random Forest Data Mining Algorithms for Microarray Data Analysis", International Journal on Advanced Electrical and Computer Engineering (IJAECE), ISSN(Online): 2349-9338, ISSN(Print): 2349932X Volume -3, Issue -4, 2016. 
[15]Decencière et al., (2014), 'Feedback on a Publicly Distributed Database: The Messidor Database', Image Analysis \& Stereology, v. 33, n. 3, p. 231-234, ISSN 1854-5165. available at: http://www.ias-

iss.org/ojs/IAS/article/view/1155
[16]World Health Organization. Global report on diabetes. Geneva: World Health Organization; 2016 